\title{
TERAPIA DE REEMPLAZO HORMONAL: VENTANA DE OPORTUNIDAD
} (HORMONE REPLACEMENT THERAPY: WINDOW OF OPPORTUNITY)

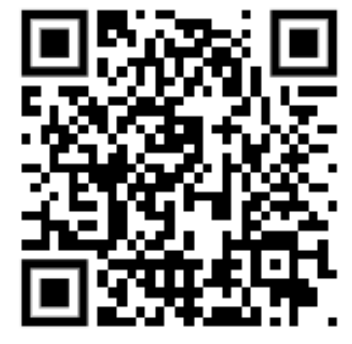

${ }^{1}$ Médico general, graduado de la Universidad Ciencias Médicas (UCIMED), médico Essity - A Leading Hygiene and Health Company, San José, Costa Rica

Código médico: 13929 carlosfonsvilla@hotmail.com

\author{
'Dr. Carlos Fonseca Villanea \\ Essity - A Leading Hygiene and Health Company, San José, Costa Rica \\ carlosfonsvilla@hotmail.com \\ https://orcid.org/0000-0002-5719-3531 \\ RECIBIDO \\ CORREGIDO \\ ACEPTADO \\ $10 / 12 / 2018$ \\ $19 / 12 / 2018$ \\ $24 / 12 / 2018$
}

\section{RESUMEN}

Se exhibirá una revisión sobre la terapia de reemplazo hormonal $(\mathrm{TRH})$, tratando de promover los criterios de "la ventana de oportunidad", con la finalidad de determinar cuáles mujeres son ideales para recibir dicha terapia de manera útil, responsable y segura. Asimismo, se contará con una explicación de la evolución desde los inicios hasta la actualidad de la TRH. Se pondrá a la vista del lector algunos principios primordiales sobre el uso adecuado de la terapia. También, se contará con una discusión sobre los beneficios de su uso sobre la salud de la mujer postmenopáusica y sus potenciales efectos secundarios. Se finalizará aclarando que la TRH utilizando los criterios de "la ventana de oportunidad" es una manera de asegurar una vida digna para las mujeres postmenopáusicas, sabiendo que en la actualidad no existe una TRH ideal, es y será un tema polémico con múltiples aristas.

PALABRAS CLAVES: Terapia de reemplazo de hormonas, estrógenos, postmenopausia, climatoterapia.

\section{ABSTRACT}

It exhibits a review on the Hormone Replacement Therapy (HRT), intending to promote the criteria on the "window of opportunity", with the intention to determine which women are ideal to receive such therapy, on a useful, responsible and safe way. As well, it explains the evolution since the beginning up to date on the hormonal replacement therapy. It is intended, for the reader, to acknowledge some of the principals on the correct use of the therapy. Also, includes a discussion on the 
benefits on the health of postmenopausal women and its potential side effects. Concluding with an asseveration, that the HRT using the criteria of "window of opportunity" is a way to assure a dignify life for postmenopausal women, knowing that to date where is no ideal HRT, is and will be a controversial topic with many sides.

KEYWORDS: Hormone replacement therapy, estrogens, postmenopause, climacteric.

\section{INTRODUCCIÓN}

La menopausia es un evento fisiológico en la vida de toda mujer, se puede entender como el final de los ciclos menstruales y el agotamiento total de la reserva ovárica. Dicho evento puede ocurrir alrededor de los 50 a 52 años de edad (1).

Existen múltiples factores los cuales las mujeres pueden tener una menopausia prematura, ya sea una insuficiencia ovárica prematura, radiación como tratamiento de cáncer pélvico o una ooforectomía en mujeres con predisposición genética de cáncer ovárico (2).

Posterior a los doce meses de la última menstruación o inmediatamente posterior a una ooforectomía bilateral se establece la postmenopausia y se instaura un estado hipoestrogénico. En este periodo, producto al descenso de los niveles séricos de estrógenos; la mujer inicia con una afección potencial en el sistema cardiovascular, genitourinario, esquelético, tegumentario y cognitivo (3). La mayoría de las féminas postmenopáusicas reportan síntomas vasomotores, mayor incomodidad, menor placer sexual y depresión que pueden afectar la calidad de vida (2). TRH es la manera de compensar la deficiencia estrogénica (4). Gracias a importantes estudios realizados en las últimas décadas se inició una controversia sobre los beneficios y potenciales riesgos de la $\mathrm{TRH}$. La "ventana de oportunidad" es una idea que se estableció posteriormente al ensayo clínico WHI en el junio 2002, dicho concepto trata de determinar cuáles son las mujeres ideales para recibir TRH (5). Según los criterios para recibir $\mathrm{TRH}$ toda mujer debe ser menor de 60 años, menos de 10 años de haber iniciado la menopausia y no presentar contraindicaciones para su uso.

El uso de la "ventana de oportunidad" reduce la incidencia de enfermedades cardiovasculares y la mortalidad femenina global (6).

\section{ANTECEDENTES HISTÓRICOS}

La historia de TRH asociado al uso de sustitución con estrógenos en mujeres climatéricas, se inició en Estados Unidos en los años 40s' (7).

Esto ocurre por influencia del libro titulado "Femenine forever", quien su autor fue Robert A. Wilson. Lo que planteaba Wilson era la posibilidad de desarrollar estrógenos conjugados provenientes de la orina de las yeguas preñadas y así mejorar la vida de todas las mujeres. [6] Al comenzar a evidenciarse los efectos a corto plazo sobre la calidad de vida de la mujer, se 
utilizó sin ninguna discriminación. "¡Estrógenos para todas y por toda la vida!" (7).

Después de treinta años de uso indiscriminado, en los años 70s' se publican estudios sobre la posibilidad de riesgo de hiperplasia uterina y cáncer endometrial (8).

En dicho momento se descubrió, que cuando se asocia un progestágeno permite revertir el riesgo de hiperplasia uterina y cáncer endometrial.

El empleo de estrógenos junto con progesterona dio origen al concepto de terapia de reemplazo hormonal combinada o más conocida como simple terapia de reemplazo hormonal . (7). Se evidenció, entre más alta la dosis de progestina menor era el riesgo de desarrollar patología endometrial; periodo después se documenta, que al aumentar la dosis de protestona aumenta proporcionalmente el riesgo cardiovascular, produciendo un efecto antagónico al estrógeno como cardioprotector (7).

A mediados de los años 90s' se publican dos estudios prospectivos, aleatorios, doble ciego y controlado con placebo que revolucionaron lo que se conocía de la TRH, estos fueron el:

- HERS I

- HERS II

Así como el WHI, en estos no se demostró ningún tipo de beneficio de la $\mathrm{TRH}$ en relación con la enfermedad cardiovascular, además se demostró un riesgo importante en el desarrollo del cáncer de mama y mayor incidencia de eventos cerebro vasculares en mujeres quienes iniciaron la TRH en el climaterio tardío; a partir de ese momento se empezó a discutir sobre la "ventana de oportunidad" para terapia de remplazo hormonal (3).

\section{PRINCIPIOS PRIMORDIALES}

La ventana de oportunidad es un concepto para recordar a los servidores en salud cuando una mujer es apta para recibir $\mathrm{TRH}$, de manera responsable y segura. La "ventana de oportunidad", como anteriormente se mencionó, debe cumplir varios requisitos, entre los que se destaca: mujeres menores de 60 años, menos de 10 años de haber iniciado la menopausia y sin contraindicaciones. Entre las contraindicaciones absolutas se encuentran (1):

- Sangrado vaginal anormal no diagnosticado

- Cáncer de mama conocido, sospechado 0 antecedentes personales del mismo

- Neoplasia conocida o sospechada dependiente de estrógenos

- Trombosis venosa profunda activa, embolia pulmonar o antecedentes de estos padecimientos

- Enfermedad tromboembolia arterial (infarto de miocardio, accidente cerebro vascular)

- Alteraciones o patologías hepáticas

Existe información evidenciada, donde se indica, si se cumple con los criterios de la "ventana de oportunidad" la mujer llega alcanzar un incremento en años de vida ajustado por calidad (QALY) por arriba de un periodo de 5 a 30 años en mujeres que iniciaron en una menopausia temprana comparada con quienes no utilizaron ninguna terapia (6). 
En las nuevas recomendaciones sobre el uso de $\mathrm{TRH}$, recomiendan que sea una terapia individualizada, ajustada a los síntomas, necesidad de prevención y expectativas de la mujer (9).Se debe valorar la historia tanto personal como familiar. Se prefiere reservar la terapia para mujer altamente sintomáticas, y limitar su uso al "menor tiempo necesario" y a la "mínima dosis efectiva" (5). La TRH debe ser una estrategia global, debe incluir por parte del proveedor de salud recomendaciones sobre estilos de vida saludable relacionados con la alimentación, ejercicio, evitar el fumado y el consumo excesivo del alcohol (3).

Antes de iniciar TRH el médico debe asegurarse que la paciente tenga claro los beneficios y posibles riesgos. El encargado de salud debe tener una clara indicación para comenzar con la $\mathrm{TRH}$, ya sea por síntomas o efectos físicos causado por la deficiencia estrogénica que afecten la calidad de vida de la paciente (1) .Se debe indagar profundamente en mujeres distraídas o con poca coherencia sobre sus síntomas, para tener un panorama más amplio de como la $\mathrm{TRH}$ puede beneficiar su calidad de vida (10).

La mujer que utilice TRH debe tener mínimo una cita por año, donde incluya una exploración física, actualización de antecedentes tanto personales como familiares, evaluación de laboratorios e imágenes, reforzamiento sobre estilo de vida saludable $y$ estrategias para prevenir enfermedades crónicas. No se requiere un aumento en el tamizaje con mamografía o citología cervical en mujeres usuarias de la terapia (5).

En términos generales, se debe agregar un progestágeno a la terapia estrogénica en toda mujer que no tenga antecedentes de histerectomía previa. Es importante recalcar que existen diferentes progestágenos que más allá de su efecto endometrial, puede tener efectos tanto beneficiosos como perjudiciales asociados; se debe escoger la progesterona dependiendo del cuadro clínico que presenta la paciente (3).

Cuando consulten paciente con síntomas únicamente asociados atrofia vulvovaginal a causa de deficiencia estrogénica, se debe escoger una terapia tópica; esta puede ser absorbida sistémicamente, pero no se requiere adjuntar un progestágeno ya que no logra estimular el endometrio. Es importante recalcar, los progestágenos vaginales o los liberados desde un dispositivo intrauterino pueden cumplir la función de los sistémicos sin los efectos secundarios conocidos (5).

\section{BENEFICIOS}

Como es conocido, la TRH es eficaz para el manejo de los síntomas vasomotores y síntomas vaginales asociados a la atrofia urogenital por efecto del estado hipoestrogénico. Se ha documentado que la $\mathrm{TRH}$ se asocia a mejoría en síntomas osteoarticulares y osteomusculares, así como, estado de ánimo, trastornos del sueño y disfunción sexual (8).

\section{- Osteoporosis postmenopáusica}

La TRH es un método eficaz para prevenir pérdidas óseas asociadas a la menopausia y disminuir la incidencia de fracturas osteoporóticas, incluyendo fracturas vertebrales y de cadera. Es segura en mujeres postmenopáusicas menores a los 60 años y sin cursar 10 
años de menopausia, el concepto de "ventana de oportunidad" también debe tenerlo en cuenta los servidores de salud antes de tomar la decisión de indicarle $\mathrm{TRH}$ para prevenir fracturas en mujeres postmenopáusicas, por los efectos secundarios que dicha terapia conlleva. Si se desea continuar la terapia posterior a los 60 años, únicamente con la meta de prevenir las fracturas osteoporóticas, se debe considerar todos los efectos a largo plazo, el método de administración y su real beneficio sobre medicamentos antiresortivos (5).

Se conoce que la $\mathrm{TRH}$ reduce el riesgo de fracturas mientras se mantenga el tratamiento, a la hora de suspenderlo este efecto protector decrece considerablemente, es importante valorar antes de suspender la terapia el riesgo de fracturas, de persistir aumentado, se debe implementar una terapia adicional comprobada de preservación ósea (8).

Aun siendo la $\mathrm{TRH}$ efectiva para prevenir fracturas producto de la osteoporosis, no se debe recomendar generalizadamente para prevenir dicha enfermedad, se debe preferir como primera línea los medicamentos antiresortivos (11).

\section{- Efecto cardiovascular}

Es un hecho bien conocido que las mujeres en etapa premenopáusica presentan una incidencia menor de infartos de miocardio que sus semejantes masculinos y llegan a igualarse al momento de la postmenopausia, esto es atribuido al bien conocido efecto cardioprotector que los estrógenos poseen.

El perfil lipídico de una mujer premenopáusica se caracteriza por tener niveles más elevados de HDL-C y niveles de LDL-C más bajos en comparación con los hombres de su misma edad. Al momento de la postmenopausia esto se invierte (niveles bajos de HDL-C y niveles más elevados de LDL-C), la terapia con estrógenos exógenos ha demostrado que revierte estos cambios en el perfil lipídico en las mujeres postmenopáusicas, así disminuyendo la formación de placas de ateroma (8).

Se debe recalcar que en ciertos estudios anteriores (WHI y HER2) donde se colocó a la progesterona como un antagonista del efecto cardioportector del estrógeno, en dichos se utilizó medroxyprogesterona; en estudio recientes se ha documentado que el uso de acetato de noretindrona $u$ otra progesterona natural no interfiere con el efecto protector a nivel cardiovascular del estrógeno; dicho esto no se debe generalizar a todas las progestinas como causantes de mayor incidencia de enfermedades cardiovasculares (11).

La evidencia de los estrógenos como cardioprotector únicamente se ha documentado si se cumple el concepto de "ventana de oportunidad". En las mujeres menores de 60 años, recientemente ingresadas en el periodo de menopausia y sin evidencia de enfermedad coronaria $(u$ otra contraindicación) (5), la TRH no causa daño endotelial temprano y hasta puede reducir la morbilidad y mortalidad por enfermedad coronaria (12). Si se decide continuar con la TRH más allá de los 60 años se tiene que analizar los riesgos y beneficios con mayor cautela (11).

Se conoce que el criterio con más importancia es la edad de inicio de la $\mathrm{TRH}$, ya que las mujeres quienes inician 
la TRH posterior a 10 años de postmenopausia, se asocia con un riesgo aumentado de eventos coronarios, principalmente en los 2 primeros años de uso (12).

Hay que insistir que no se recomienda el inicio de la TRH en mujeres mayores de 60 años con el único objetivo de la prevención primaria de enfermedad cardiovascular (5).

\section{- Otros beneficios}

Existen beneficios adicionales en la $\mathrm{TRH}$; el uso de estrógenos tópicos para mantener la salud del tracto urogenital y corregir los cambios epiteliales a causa del estado hipoestrogenico es la indicación más utilizada para la TRH. En general, la TRH presenta beneficios en el tejido conectivo, articular, piel y en la estructura de los discos intervertebrales en las usuarias de dicha terapia. Se cree que el uso de TRH por más de 4 años puede tener un papel en la prevención del cáncer de colon, disminuir el riesgo de enfermedades cognitivas y mejorar la calidad del sueño; aunque por ahora existe poca evidencia (8).

Actualmente, gracias las nuevas tecnologías, conocimientos en diagnóstico y cribado oncológico, además asociado los estudios genéricos; es común encontrar mujeres en periodo premenopáusico a quienes se le realizaron salpingo-ooforectomía (RRSO), induciendo una menopausia iatrogénica (2). Existen numerosas causas por la cual se indica una RRSO, puede ocurrir al momento de la histerectomía para tratar una patología ovárica o en casos donde la mujer tiene una predisposición genética de padecer cáncer de mama o cáncer de ovario (13). Está documentado que esta población se puede beneficiar con la TRH en el primer año posterior a la RRSO, de esta manera evitando trastornos sexuales y endocrinológicos. El servidor de salud puede recomendar $\mathrm{TRH}$ previo a la cirugía $(2,14)$.

Producto de la RRSO, las mujeres premenopáusicas en ocaciones presentan trastornos sexuales que las afectan durante su vida, como la

disminución del placer, resequedad vaginal, malestar al momento de la actividad sexual y con esto menor frecuencia de sus actividades sexuales. La TRH es una herramienta que ayuda a combatir estas molestis. las mujeres premenopáusicas con RRSO sufren síntomas menopaúsicos más intensos y frecuentes comparadas con las mujeres con menopausia fisiológica (13). Siempre individualizando, conociendo la causa que produjo la RRSO (4), si se decide indicar la TRH se debe recomendar el uso hasta los 51.5 años de edad (13).

\section{POTENCIALES EFECTOS ADVERSOS}

A lo largo de la historia se le han responsabilizado diferentes patologías al uso de la $\mathrm{TRH}$, como por ejemplo el cáncer de mama, de endometrio, tromboembolismo venoso, accidentes vasculares cerebrales y coronarios han sido los más estudiados.

\section{- Cáncer de mama}

Al estudiar la relación entre el cáncer de mama y la $\mathrm{TRH}$ existen problemas epidemiológicos, la incidencia de cáncer de mama es diferente según la población analizada; los datos conocidos que están 
disponibles no pueden ser aplicables en todas las mujeres por igual (9).

La relación entre el cáncer de mama y $\mathrm{TRH}$ persiste siendo un tema controversial.

Para entender el riesgo de cáncer de mama y la TRH primero se tiene que dividir a las mujeres en dos grupos:

1. mujeres con útero (requieren progesterona)

2. mujeres histerectomizadas.

En mujeres histerectomizadas, en las cuales únicamente requieren estrógenos conjugados, se ha documentado que estos no aumentan el riesgo de cáncer de mama y hasta puede tener un efecto protector. Si se utilizan los criterios de la "ventana de oportunidad" la aparición de cáncer de mama está más relacionada al asociar estrógeno-progesterona que al estrógeno únicamente $(9,14)$.

En el grupo de las mujeres con útero conservado, se tiene que agregar una progestina para evitar el riesgo de hiperplasia endometrial o cáncer de endometrio como es conocido, se ha estudiado que al agregar una progestina al estrógeno de base puede aumentar levemente el riesgo de cáncer de mama (menos de $0.1 \%$ anual, o una incidencia $<1,0$ cada 1000 mujeres por año de uso) (5). Cabe rescatar, este riesgo es menor al asociado a factores del estilo de vida, como la obesidad, consumo de alcohol o sedentarismo (9).

Actualmente no existen datos para valorar las diferencias entre dosis, vía de administración, progesterona natural o progestágenos, que relacionen una mayor o menor incidencia de cáncer de mama. No obstante, se están realizando estudios sobre el uso de diferentes progestágenos para poder utilízarlos por largo tiempo sin aumentar el riesgo de cáncer de mama (9).

La relación entre la TRH y el cáncer de mama presenta muchas opiniones, es un tema muy discutido. Siempre se debe individualizar a cada mujer para valorar los pros y contras.

\section{- Cáncer de endometrio}

De forma general, en las últimas dos décadas el cáncer endometrial es el carcinoma ginecológico más frecuente en países desarrollados, es el padecimiento más frecuente en la mujer postmenopáusica, pero hasta un $25 \%$ de los diagnósticos se realizan en el periodo perimenopausico y un 5\% ocurre antes de los 40 años (15).

Como se comentó previo, las mujeres quienes conservan el útero presentan un riesgo de hiperplasia endometrial y cáncer de endometrio cuando se administra estrógenos exógenos sin oposición con progesterona (8).

Actualmente, siempre se recomienda a la fémina quien conserva su útero un esquema de estrógeno-progesterona, ya que se asocia a una menor incidencia de hiperplasia endometrial y cáncer de endometrio en la población general (12).

Se conoce que los dispositivos intrauterinos liberadores de levonogestrel han presentado ventajas y cumplen la función de los progestágenos orales. En la actualidad, existen regímenes que contienen niveles bajos o ultra-bajos de estrógenos y progesterona, estos han demostrado que reducen el riesgo de hiperplasia endometrial y el sangrado vaginal (5). 
- Tromboembolismo y eventos cardiovasculares

El riesgo de tromboembolismo venoso grave relacionado a $\mathrm{TRH}$ se debe ya que los estrógenos exógenos aumentan la producción de factores de coagulación y la resistencia para activar la proteína $\mathrm{C}$ (15).

También se puede utilizar el concepto de "ventana de oportunidad" porque se encuentra muy relacionado con la edad;

se observa raramente en pacientes menores de 60 años (5). El troembolismo también se relaciona con estilos de vida inadecuados, como es la obesidad y fumado; además de otros padecimientos como la trombofilia (12). Los estrógenos transdérmicos pueden disminuir el riesgo porque evitan el primer paso metabólico a nivel hepático (12).

Los eventos cardiovasculares también están muy relacionados a la edad, por esto es importante tener claro el concepto de "ventana de oportunidad", es poco probable que ocurra accidente cerebro vascular relacionado a la $\mathrm{TRH}$ antes de los 60 años (5).

\section{CONCLUSIONES}

La TRH ha sido, es y seguirá siendo un tratamiento polémico y controvertido, es importante valorar, que gracias a los nuevos aportes de la tecnología médica las mujeres van a vivir un tercio de su vida en el periodo de menopausia (11). La TRH es una manera de asegurar una vida digna en las mujeres; siempre bajo la tutela de un servidor de salud experto y siguiendo las pautas de la "ventana de oportunidad". No existe en este momento una TRH ideal. Cualquier TRH va tener beneficios y no van a estar exentos de riesgos. Lo más importante es siempre individualizar cada caso. Va ser un tema que se seguirá estudiando y se debe estar atento a las innovaciones del futuro.

\section{REFERENCIAS}

1. Fritz MA, Speroff L. Endocrinología Ginecológica Clínica y Esterilidad. 8 Ed.. Lippincott Williams \& Wilkins; 2011

2. Vermeulen RF, Beurden MV, Kieffer JM, Bleiker EM, Valdimarsdottir HB, Massuger LF, Mourits MJ, Gaarenstroom KN, van Dorst EB, van der Putten HW, Aaronson NK. Hormone replacement therapy after risk-reducing salpingo-oophorectomy minimises endocrine and sexual problems: A prospective study. European Journal of Cancer. 2017 Oct;84:159-167. https://doi.org/10.1016/j.ejca.2017.07.018

3. Clinical Guideline on Management of Menopausal Symptoms.www.aafp.org/afp/2014/0901/p338.html\#. Accessed 2014 Septiembre 01.

4. Johansen N, Liavaag AH, Tanbo TG, Dahl AA, Pripp AH, Michelsen TM. Sexual activity and functioning after risk-reducing salpingo-oophorectomy: Impact of hormone replacement therapy. Gynecologic Oncology. 2016 01;140(1):101-106. https://doi.org/10.1016/j.ygyno.2015.11.016 
5. Sturdee DW, Pines A. Updated IMS recommendations on postmenopausal hormone therapy and preventive strategies for midlife health. Climacteric. 201105 13;14(3):302-320. https://doi.org/10.3109/13697137.2011.570590

6. Hodis HN, Collins P, Mack WJ, Schierbeck LL. The timing hypothesis for coronary heart disease prevention with hormone therapy: past, present and future in perspective. Climacteric. 201205 22;15(3):217-228. https://doi.org/10.3109/13697137.2012.656401.

7. Campodónico I. Historia crítica de las terapias de reemplazo hormonal. Una visión particular después del estudio WHI. Rev. Obstet. Ginecol.- Hosp. Santiago Oriente Dr. Luis Tisné Brousse. 2018;13(01):51-53.

8. Hsia J. Conjugated Equine Estrogens and Coronary Heart Disease. Archives of Internal Medicine. 200602 13;166(3):357. https://doi.org/10.1001/archinte.166.3.357

9. Effects of Conjugated Equine Estrogen in Postmenopausal Women With Hysterectomy. JAMA. 200404 14;291(14):1701. https://doi.org/10.1001/jama.291.14.1701

10. Jalava-Broman J, Mäkinen J, Sillanmäki L, Vahtera J, Rautava P. Characteristics associated with initiation of hormone replacement therapy among Finnish women: A register-linkage study. Maturitas. 2016 07;89:73-78. https://doi.org/10.1016/j.maturitas.2016.04.011

11. Agarwal S, Alzahrani F, Ahmed A. Hormone Replacement Therapy: Would it be Possible to Replicate a Functional Ovary?. International Journal of Molecular Sciences. 2018 Oct 14;19(10):3160. https://doi.org/10.3390/ijms19103160

12. Toh S. Coronary Heart Disease in Postmenopausal Recipients of Estrogen Plus Progestin Therapy: Does the Increased Risk Ever Disappear?. Annals of Internal Medicine. 201002 16;152(4):211. https://doi.org/10.7326/0003-4819-152-4-201002160-00005

13. Sarrel PM, Sullivan SD, Nelson LM. Hormone replacement therapy in young women with surgical primary ovarian insufficiency. Fertility and Sterility. 2016 Dec;106(7):1580-1587. https://doi.org/10.1016/j.fertnstert.2016.09.018

14. Angioli R, Luvero D, Armento G, Capriglione S, Plotti F, Scaletta G, Lopez S, Montera R, Gatti A, Serra GB, Benedetti Panici P, Terranova C. Hormone replacement therapy in cancer survivors: Utopia?. Critical Reviews in Oncology/Hematology. 2018 04;124:51-60. https://doi.org/10.1016/j.critrevonc.2018.02.005

15. O'Donnell RL, Clement KM, Edmondson RJ. Hormone replacement therapy after treatment for a gynaecological malignancy. Current Opinion in Obstetrics and Gynecology. 2016 02;28(1):3241. https://doi.org/10.1097/gco.0000000000000238 